uber dem Boden und wiederholte die Versuche im October zu Laqueue auf dem Plateau von la Brie.

In einer spätern Arbeit werde ich angeben, wclche wichtige Rolle der active atmosphärische Sauerstoff bei den Erscheinungen der Nitrification spielt. Ohne allen $Z$ weifel rühren von diesen, wenigstens zum Theil, die Anomalien her, welche in letzterer Zeit Cloëz, de Luca und Boussingault hinsichtlich des Gehalts der Luft an Nitraten beobachtet haben. Schönbein hat gezeigt, dass der Stickstoff der Atmosphäre unter dem Einfluss von Ozon und einer alkalischen Base in ein Nitrat übergehen kann; ich habe andererseits gefunden, dass das freie oder das kohlensaure Ammoniak gleichfalls durch Ozon und bei $A b w e s e n h e i t$ von Stickstoffgas, eine nicht weniger auffallende Nitrification erleidet. Es wird also künftighin bei Untersuchungen uber die Quelle der in der Natur vorkommenden Nitrate vorzüglich auf das Ammoniak und den activen Sauerstoff Rücksicht zu nehmen sein.

\title{
XI
}

Ueber einen eigenthümlichen Fall, in welchem Baryterde durch Schwefelsäure nicht oder doch nicht vollständig gefällt wird.

Von

\section{Th. Scheerer.}

Der im Handel vorkommende Borax pflegt durch kleine Mengen von schwefelsaurem Natron und Chlornatrium verunreinigt $\mathrm{zu}$ sein; und auch im geschmolzenen Borax (Boraxglas) lassen sich diese Beimengungen nachweisen. Eine gleiche Verunreinigung zeigt das (bei Löthrohr-Versuchen angewendete) sogenannte Phosphorsalz, phosphorsaures Natron-Ammoniak; wird dasselbe aber geschmolzen, so lässt sich in dem zurückbleibenden meta- 
phosphorsauren Natron, $\mathrm{Na}^{a} \mathbf{P}$, wohl noch Chlor, aber durch eine Baryterae-Solution keine Schwefelsäure mehr entdecken.

Es erschien mir nun unwahrscheinlich, dass, wahrend ein kleiner Schwefelsäuregehalt aus schmelzendem borsauren Natron nicht ausgetrieben wird, ein ähnlicher Schwefelsäuregchalt aus schmelzendem metaphosphorsauren Natron ausgetrieben werde. Ich kam daher auf die Vermuthung, dass auch letzteres noch Schwefelsäure enthalte, dass aber die Gegenwart der Metaphosphorsüure die Fällung der Schwefelsäure durch eine Baryterde-Solution beeinträchtige. Diess bat sich bestätigt.

Setzt man zu einer Auflösung von metaphosphorsaurem Natron eine grössere Menge verdünnte Salzsäure und darauf Chlorbaryum-Solution - welche man tropfenweise und unter Umrühren hinzufügt, damit sich der anfangs entstehende Niederschlag von metaphosphorsaurem Baryt in einem hinreichenden Ueberschuss an Salzsäure und Wasser wieder auflöst -- und giesst dann in die vollkommen klare Lösung stark verdünnte Schwefelsäure, so entsteht kein Niederschlag von schwefelsaurem Baryt. Nach mehrstündigem, mitunter erst nach tagelangem Stehen, fängt die Flüssigkeit an, sich zu trüben; wird sie dagegen gekocht, so hat diess die Bildung eines mehr oder weniger starken weissen Niederschlages zur Folge.

Durch Wiederholungen dieses Versuchs überzeugte ich mich, dass es beim Hervorufen dieser paradoxen Erscheinung auf die Verdünnungsverhältnisse ankommt. Ist die Schwefelsäure nicht hinreichend verdünnt, oder setzt man ein sehr grosses Uebermaass verdünnter Schwefelsäure hinzu, so wird dadurch die Bildung jenes weissen Niederschlags beschleunigt; aber doch scheidet sich derselbe meist nur allmählich aus, und die davon abfiltrirte Flüssigkeit fährt fort sich zu trüben. Auch pflegt der Niederschlag nicht das gewöhnliche Ansehn des aus Solutionen präcipitirten schwefelsauren Baryts zu haben, sondern sich zum Theil an den Wänden des Glases in mikroskopisch kleinen Krystallen ahzusetzen. 
Weder mit gewöhnlicher - dreibasischer - Phosphorsäure noch mit Pyrophosphorsäure konnten ähnliche Erscheinungen hervorgerufen werden.

Es war nun von Interesse, sowohl hinsichtlich jener Verdünnungsverhältnisse, wie über die chemischc Beschaffenheit des eigenthümlichen Niederschlages, etwas Näheres zu ermitteln. Diess unternahm mein Assistent, Herr Dr. Rube, indem er die folgenden Versuche austellte.

Die hierzu angewendeten Solutionen besassen Concentrationsgrade, wie sie in der folgenden Zusammenstellung. angegeben siud. C. C. bezeichnet einen Cubikcentimeter.

A.

Solution von metaphosphors. Natron.

In $100 \mathrm{C}$. C.
B.

Verdünnte

Salzsäure.

In 100 C. C.
C.

Solution von

Chlorbaryum.

In $100 \mathrm{C}$. C.
D.

Verdünnte

Schwefelsäure.

Bei der ersten Versuchsreihe.

$10 \mathrm{Gr} . \dot{\mathrm{N}} \mathrm{a}^{2} \ddot{\mathrm{P}} \quad 8$ C. C. concen- $10,50 \mathrm{Gr} . \mathrm{Ba} G \mathrm{l} \quad 6,32 \mathrm{Gr} . \dddot{\mathrm{S}} \dot{\mathrm{H}}$. trirte Salzsäure.

Bei der zweiten Versuchsreihe.

Desgleichen. Desgleichen. $19,96 \mathrm{Gr} . \mathrm{Ba} \in \mathrm{l}$. 26,94 Gr. S̈̈.

Um die Resultate der verschiedenen Versuche möglichst übersichtlich und leicht mit einander vergleichbal. zu machen, wurden dieselben tabellarisch zusammengestellt. Mit $A, B, C$ und $D$ sind die eben angeführten Solutionen von den angegebenen Concentrationsverhältnissen bezeichnet.

Erste Versuchsreihe.

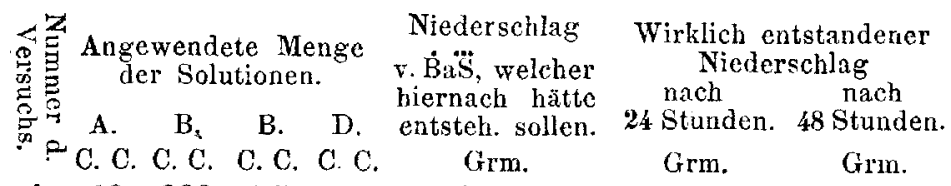

1. $10300 \quad 2,5 \quad 2, \check{3} \quad \mathbf{0 , 2 9 5}$ Spur von Stärkere

$\begin{array}{llllllll}2 . & 10 & 300 & 5 & 5 & 0,589 & \text { Trübung. } & \text { Trübung. } \\ 0.038 & 0,175\end{array}$

$\begin{array}{llllllll}3 . & 15 & 300 & 5 & 5 & 0,589 & 0,146 & 0,337\end{array}$

$\begin{array}{llllllll}4 . & 5 & 300 & 5 & 5 & 0,589 & 0,269 & 0,482\end{array}$

Aus der Vergleichung des Versuchs 2 mit den Versuchen 4 und 3 ergiebt sich, dass hier die Menge des Niederschlags nicht nur bei der Verminderung des phos- 
phorsauren Natrons, sondern auch bei der Vermehrung desselben zunimmt. Jedoch haben alle diese Niederschläge eine verschiedene chemische Zusammensetzung, und die darin enthaltenen Mengen der Baryterde verhalten sich nicht wie die Gewichte der Niederschläge. Die beim Versuche 1 entstandenen Trübungen waren zu gering, um sie quantitativ genau zu bestimmen.

Zweite Versuchsreihe.

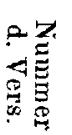

Angewendete Menge der Solutionen.

A. B.

C.

D.

C. C. C. C. C. C. C. C. Grm. Grm.

Niederschlag von $\dot{\mathrm{B}} \mathrm{a} \mathrm{S}$, welcher hier nach hätte entstehen sollen.
Wirklich ertstandener Niederschlag nach 24 St.

$\begin{array}{rrrrrrr}5 . & 20 & 300 & 5 & 5 & 1,119 & 0,976 \\ 6 . & 25 & 300 & 5 & 5 & 1,119 & 0,622 \\ 7 . & 30 & 300 & 5 & 20 & 1,119 & 0,781\end{array}$

Bei der näheren Untersuchung der Niederschläge beider Versuchsreihen fand Herr Dr. Rube, dass dieselben sämmtlich nicht bloss schwefelsauren, sondern auch phosphorsauren Baryt enthielten. Die Menge der Phosphorsäure variirte in einigen derselben zwischen 6 p. C. und 14 p. C.

Es lag nicht die Absicht vor, die quantitative Seite dieses eigenthümlichen Verhaltens der Schwefelsäure bei Gegenwart ron Metaphosphorsäure genau zu erforschen - wobei jedenfalls auch die Temperaturen der Solutionen hätten in Berücksichtigung kommen müssen - sondern nur die Thatsache anschaulich zu machen, dass in diesem Fall die gewohnte Unfehlbarkeit der gegenseitigen Fällung von Baryterde und Schwefelsäure eine Ausnahme erleidet.

Nach Dr. Rube's Versuchen zeigen Strontianerde und - was weniger befremden kann - Kalkerde dasselbe Verhalten zur Metaphosphorsäure wie Baryterde; auch überzeugte sich derselbe, dass metaphosphorsaures Natron in ganz ähnlicher Weise die Ausfällung der Baryterde, Strontianerde und Kalkerde mittelst kohlensauren Ammoniaks beeinträchtigt. 